\title{
Schwarz alternating methods for anisotropic problems with prolate spheroid boundaries
}

\author{
Zhenlong Dai ${ }^{1}$, Qikui Du ${ }^{1 *}$ and Baoqing Liu ${ }^{2}$
}

*Correspondence:
duqikui@njnu.edu.cn
1 Jiangsu Key Laboratory
for NSLSCS, School
of Mathematics Sciences,
Nanjing Normal University,
No. 1 Wenyuan Road,
Nanjing 210023 , People's
Republic of China
Full list of author information
is available at the end of the
article

*Correspondence: duqikui@njnu.edu.cn

Jiangsu Key Laboratory for NSLSCS, School

of Mathematics Sciences,

Nanjing Normal University,

Nanjing 210023, People's

Republic of China

is available at the end of the

\begin{abstract}
The Schwarz alternating algorithm, which is based on natural boundary element method, is constructed for solving the exterior anisotropic problem in the threedimension domain. The anisotropic problem is transformed into harmonic problem by using the coordinate transformation. Correspondingly, the algorithm is also changed. Continually, we analysis the convergence and the error estimate of the algorithm. Meanwhile, we give the contraction factor for the convergence. Finally, some numerical examples are computed to show the efficiency of this algorithm.
\end{abstract}

Keywords: Schwarz alternating algorithm, Exterior anisotropic problem, Prolate ellipsoidal, Artificial boundary, Iteration method

\section{Background}

How to deal with boundary value problems has always been a essential part of partial differential equation. Finite difference method (FDM) (Evans 1977) and finite element method (FEM) (Brenner and Scott 1996) are the most widely used method to solve PDE numerically. These two methods become in vain when it comes to the problem over unbounded domain. To overcome this, boundary element method (BEM), which can reduce the dimension of the computational domain and is suitable for solving problems in the unbounded domains, is proposed in Feng (1980). Although, it is better to handle BEM with infinite regions, it doesn't work so well as FEM in bounded ones. Hence, the coupling of BEM and FEM becomes the interest of researchers. Papers MacCamy and Marin (1980), Hsiao and Porter (1986), Wendland (1986), Costabel (1987), Han (1990) had focused on this method. In 1983, Feng firstly proposed a direct and natural coupling method. Later in the same year, Feng and Yu (1983) formally named the method as natural boundary element method (NBEM). Meanwhile, the DtN method, which has the similar principle with NBEM, is proposed in Keller and Givoli (1989), Grote and Keller (1995). Du and $\mathrm{Yu}$ (2001), Hu and Yu (2001), Gatica et al. (2003), Koyama (2007), Koyama (2009), Das and Mehrmann (2016), Das and Natesan (2014), Das (2015) and references therein present the applications of this methods. 
Among the reasons that effects the NBEM, the shape of artificial boundary is the essential one. Classically, circle (Givoli and Keller 1989) and spherical (Grote and Keller 1995; Wu and Yu 1998, 2000a) are chosen as the artificial boundaries. Few papers Grote and Keller (1995), Wu and Yu (2000b), Huang and Yu (2006) focus on the special artificial boundaries. These papers also proved the classic artificial boundaries were not suitable for the problem with irregular shape. On the other hand, the coupling of FEM and BEM are not enough as the performance of computer developed. The domain decomposition method (DDM) (Brenner and Scott 1996), which separates the infinite region as sum of bounded one and unbounded one with an artificial boundary on which an iteration method is constructed in, is applied on the NBEM (Yu 1994). Wu and Yu (2000b) applied this method over an infinite region. Continually, Huang et al. (2009) and Luo et al. (2013) applied this method in different problems.

In this paper, we consider the anisotropic harmonic problem over an exterior threedimensional domain. A Schwartz alternating method is designed for the numerical solution with prolate artificial boundaries.

The outline of the paper is as follows. In "Schwarz alternating algorithm based on NBR" section, we divide the original domain $\Omega$ into two overlapping subdomains $\Omega_{1}$ and $\Omega_{2}$ by choosing two artificial boundaries $\Gamma_{1}$ and $\Gamma_{2}$, then we construct the Schwarz alternating algorithm. We prove the convergence of the algorithm in "Convergence of the algorithm" section. The convergence rate of the algorithm is analysed in the "Analysis of the convergence rate" section. In "The error estimates of the algorithm" section, we deduce the error estimates of the discrete algorithm. In "Numerical results" section, numerical examples are computed to express the advantages of this method. Finally, we give some conclusions in "Conclusions" section.

\section{Schwarz alternating algorithm based on NBR}

Let $\Omega \subset R^{3}$ be a cuboid Lipschitz unbounded domain and $\Gamma_{0}=\partial \Omega$ is its boundary. We consider the following exterior Dirichlet problem

$$
\begin{cases}-\left(K_{1} \frac{\partial^{2}}{\partial x^{2}}+K_{1} \frac{\partial^{2}}{\partial y^{2}}+K_{2} \frac{\partial^{2}}{\partial z^{2}}\right) u=0, & \text { in } \Omega, \\ u=g, & \text { on } \Gamma_{0}, \\ u \rightarrow 0 & \text { as } r \rightarrow \infty,\end{cases}
$$

where $K_{1}$ and $K_{2}$ are two different anisotropic parameters, $g$ is a given function that satisfies $g \in H^{1 / 2}\left(\Gamma_{0}\right)$, and $r=\sqrt{x^{2}+y^{2}+z^{2}}$. The third item of Eq. (1) keeps the existence and uniqueness of the solution.

Let $\Gamma_{1}=\left\{(x, y, z): \frac{x^{2}+y^{2}}{d^{2}}+\frac{z^{2}}{c^{2}}=1, c>d>0\right\}$ and $\Gamma_{2}=\left\{(x, y, z): \frac{x^{2}+y^{2}}{b^{2}}+\frac{z^{2}}{a^{2}}=1\right.$, $a>b>0\}$ denote two artificial prolate spheroids. For clarity, we must mention that $d>b$ and $c>a$. This means that $\Gamma_{2}$ is totally inside $\Gamma_{1}$. Define $\Omega_{2}$ as the unbounded domain outside the boundary $\Gamma_{2}$ and $\Omega_{1}$ be a bounded domain between $\Gamma_{0}$ and $\Gamma_{1}$ (see Fig. 1).

According to DDM (Brenner and Scott 1996), we construct the Schwarz alternating method as follows: 


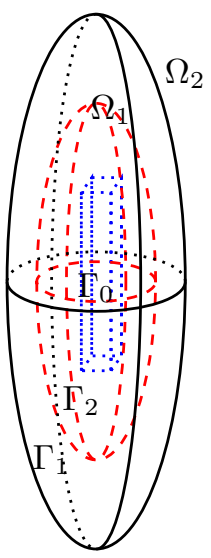

Fig. 1 Domain participation

$$
\begin{cases}-\left(K_{1} \frac{\partial^{2}}{\partial x^{2}}+K_{1} \frac{\partial^{2}}{\partial y^{2}}+K_{2} \frac{\partial^{2}}{\partial z^{2}}\right) u_{1}^{(2 k+1)}=0, & \text { in } \Omega_{1}, \\ u_{1}^{(2 k+1)}=u_{2}^{(2 k)}, & \text { on } \Gamma_{1}, \\ u_{1}^{(2 k+1)}=g, & \text { on } \Gamma_{0},\end{cases}
$$

and

$$
\begin{cases}-\left(K_{1} \frac{\partial^{2}}{\partial x^{2}}+K_{1} \frac{\partial^{2}}{\partial y^{2}}+K_{2} \frac{\partial^{2}}{\partial z^{2}}\right) u_{2}^{(2 k+2)}=0, & \text { in } \Omega_{2}, \\ u_{2}^{(2 k+2)}=u_{1}^{(2 k+1)}, & \text { on } \Gamma_{2}, \\ u_{2}^{(2 k+2)} \rightarrow 0, & \text { as } r \rightarrow \infty,\end{cases}
$$

where $k=0,1, \ldots$ and $u_{2}^{(0)}=\widetilde{u}$.

Setting the initial value of function $u_{2}^{(0)}$ on boundary $\Gamma_{1}$ as $u_{2}^{(0)} \mid \Gamma_{1}=0$. Hence, we can solve the problem (2). Furthermore, with the limitation of $u_{1}^{(1)}$ on $\Gamma_{2}$, one solves the problem (3). Sequentially, we solve the problem in $\Omega_{1}$ again with substituting the value of solution $u_{2}^{(2)}$ on $\Gamma_{1}$. Then, we repeat the steps for $k=1,2, \ldots$ and so on.

By the above description, obviously, we applied FEM in the problem over $\Omega_{1}$ and BEM (Feng and Yu 1983) in $\Omega_{2}$. Before using BEM to solve problem (3), the following transformation is introduced.

$$
\left\{\begin{array}{l}
x=\sqrt{K_{1}} x_{1} \\
y=\sqrt{K_{1}} y_{1} \\
z=\sqrt{K_{2}} z_{1}
\end{array}\right.
$$

For simplicity, the corresponding signals under the coordinate system $\left(x_{1}, y_{1}, z_{1}\right)$ can be defined by adding an apostrophe on the original ones, e.g. $\Omega \rightarrow \Omega^{\prime}$. Therefore, problem (3) can be expressed as the harmonic problem according to the new coordinate system. 


$$
\begin{cases}-\left(\frac{\partial^{2}}{\partial x_{1}^{2}}+\frac{\partial^{2}}{\partial y_{1}^{2}}+\frac{\partial^{2}}{\partial z_{1}^{2}}\right) u_{2}^{(2 k+2)}=0, & \text { in } \Omega_{2}^{\prime}, \\ u_{2}^{(2 k+2)}=u_{1}^{(2 k+1)}, & \text { on } \Gamma_{2}^{\prime}, \\ u_{2}^{(2 k+2)} \rightarrow 0, & \text { as } r^{\prime} \rightarrow \infty\end{cases}
$$

We introduce the prolate spheroidal coordinates $(\mu, \theta, \varphi)$, such that $\Gamma_{2}^{\prime}$ coincides with the prolate spheroid $\mu=\mu_{2}$ and $\Omega_{2}^{\prime}=\left\{(\mu, \theta, \varphi) \mid \mu>\mu_{2}>0, \theta \in[0, \pi], \varphi \in[0,2 \pi]\right\}$.

$$
\begin{cases}x_{1}=f \sinh \mu \sin \theta \cos \varphi, & \mu \geq \mu_{2}>0, \\ y_{1}=f \sinh \mu \sin \theta \sin \varphi, & \theta \in[0, \pi], \\ z_{1}=f \cosh \mu \cos \theta, & \varphi \in[0,2 \pi],\end{cases}
$$

where $f=\sqrt{\frac{a^{2}}{K_{2}}-\frac{b^{2}}{K_{1}}}, a=f \cosh \mu_{2}$ and $b=f \sinh \mu_{2}$.

For simplicity, the problem (5) can be expressed as

$$
\begin{cases}-\Delta u=0, & \text { in } \Omega_{2}^{\prime}, \\ u=u_{1}, & \text { on } \Gamma_{2}^{\prime}, \\ u \rightarrow 0, & \text { as } r^{\prime} \rightarrow \infty .\end{cases}
$$

By the separation of variable (Zhang and Jin 1996), we have the solution of (7) as follows

$$
\begin{aligned}
u(\mu, \theta, \varphi) & =\sum_{n=0}^{\infty} \sum_{m=-n}^{n} \frac{Q_{n}^{m}(\cosh \mu)}{Q_{n}^{m}\left(\cosh \mu_{2}\right)} U_{n m} Y_{n m}(\theta, \varphi) \\
& \equiv H\left(u_{2}, \mu, \theta, \varphi\right), \quad \mu \geq \mu_{2}>0,
\end{aligned}
$$

where

$$
\begin{aligned}
U_{n m} & =\int_{0}^{2 \pi} \int_{0}^{\pi} u_{2}\left(\mu_{2}, \theta, \varphi\right) Y_{n m}^{*}(\theta, \varphi) \sin (\theta) d \theta d \varphi \\
Y_{n m}^{*} & =(-1)^{m} Y_{n m}(\theta, \varphi)=(-1)^{m} \sqrt{\frac{2 n+1}{4 \pi} \frac{(n-m) !}{(n+m) !}} P_{n}^{m}(\cos (\theta)) e^{i m \varphi}
\end{aligned}
$$

$P_{n}^{m}$ and $Q_{n}^{m}$ are the first and second kind of the associated Legendre functions. Therefore, the solution $u$ of (7) restricted on $\Gamma_{1}^{\prime}$ can be expressed as

$$
u\left(\mu_{1}, \theta, \varphi\right)=H\left(u_{2}, \mu_{1}, \theta, \varphi\right) .
$$

Similarly, we have the equivalent problem of (2). Thus, the Schwarz alternating algorithm can be expressed as follows:

$$
\begin{cases}-\Delta u_{1}^{(2 k+1)}=0, & \text { in } \Omega_{1}^{\prime}, \\ u_{1}^{(2 k+1)}=g^{\prime}, & \text { on } \Gamma_{0}^{\prime}, \\ u_{1}^{(2 k+1)}=u_{2}^{(2 k)}, & \text { on } \Gamma_{1}^{\prime},\end{cases}
$$


and

$$
\begin{cases}-\Delta u_{2}^{(2 k+2)}=0, & \text { in } \Omega_{2}^{\prime}, \\ u_{2}^{(2 k+2)}=u_{1}^{(2 k+1)}, & \text { on } \Gamma_{2}^{\prime}, \\ u_{2}^{(2 k+2)} \rightarrow 0, & \text { as } r^{\prime} \rightarrow \infty .\end{cases}
$$

where $k=0,1, \ldots$. The detail is similar to the original.

\section{Convergence of the algorithm}

We define the following spaces

$$
\begin{aligned}
& W_{0}^{1}\left(\Omega^{\prime}\right)=\left\{v \mid \frac{v}{\sqrt{1+x_{1}^{2}+y_{1}^{2}+z_{1}^{2}}} \in L^{2}\left(\Omega^{\prime}\right) ; \frac{\partial v}{\partial x_{1}}, \frac{\partial v}{\partial y_{1}}, \frac{\partial v}{\partial z_{1}} \in L^{2}\left(\Omega^{\prime}\right)\right\}, \\
& \stackrel{\circ}{W}_{0}^{1}\left(\Omega^{\prime}\right)=\left\{v \in W_{0}^{1}\left(\Omega^{\prime}\right)|v|_{\Gamma_{0}^{\prime}}=0\right\} .
\end{aligned}
$$

Solutions of (9) and (10) are in $V_{1}=H_{0}^{1}\left(\Omega_{1}^{\prime}\right)$ and $V_{2}=\stackrel{\circ}{W}_{0}^{1}\left(\Omega_{2}^{\prime}\right)$, respectively. Moreover, we denote the $\stackrel{\circ}{W}_{0}^{1}\left(\Omega^{\prime}\right)$ as $V$. Both functions of $V_{1}$ and $V_{2}$ can be extended into $V$. For example, we can extend $u \in V_{1}$ by zero in $\Omega^{\prime} \backslash \Omega_{1}^{\prime}$ to a function in $V$.

Hence, we have the equivalent variational form of (5):

$$
\begin{cases}\text { Find } w=u-\widetilde{u} \in \stackrel{\circ}{W}_{0}^{1}\left(\Omega^{\prime}\right), & \text { such that } \\ D_{\Omega^{\prime}}(w, v)=-D_{\Omega^{\prime}}(\widetilde{u}, v), & \forall v \in \mathscr{W}_{0}^{1}\left(\Omega^{\prime}\right),\end{cases}
$$

where $D_{\Omega^{\prime}}(u, v)=\int_{\Omega^{\prime}} \nabla u \cdot \nabla v \mathrm{~d} x_{1} \mathrm{~d} y_{1} \mathrm{~d} z_{1}, \quad \widetilde{u} \in W_{0}^{1}\left(\Omega^{\prime}\right)$ has compact support and $\left.\widetilde{u}\right|_{\Gamma_{0}^{\prime}}=g \cdot|u|_{1}=\sqrt{D_{\Omega^{\prime}}(u, u)}$ is an equivalent norm of $\mathscr{W}_{0}^{1}\left(\Omega^{\prime}\right)$. If $g \in H^{\frac{1}{2}}\left(\Gamma_{0}^{\prime}\right)$, then there exists $\widetilde{u}$ such that the solution of (11) exists and is uniquely determined.

Then (9) and (10) are equivalent to the following variational problems:

$$
\begin{cases}\text { Find } w_{1}^{(2 k+1)}=u_{1}^{(2 k+1)}-\left.u^{(2 k)}\right|_{\Omega_{1}^{\prime}} \in V_{1}, & \text { such that } \\ D_{\Omega_{1}^{\prime}}\left(w_{1}^{(2 k+1)}, v\right)=-D_{\Omega_{1}^{\prime}}\left(u^{(2 k)}, v\right), & \forall v \in V_{1},\end{cases}
$$

and

$$
\begin{cases}\text { Find } w_{2}^{(2 k+2)}=u_{2}^{(2 k+2)}-\left.u^{(2 k+1)}\right|_{\Omega_{2}^{\prime}} \in V_{2}, & \text { such that } \\ D_{\Omega_{2}^{\prime}}\left(w_{2}^{(2 k+2)}, v\right)=-D_{\Omega_{2}^{\prime}}\left(u^{(2 k+1)}, v\right), & \forall v \in V_{2} .\end{cases}
$$

Let

$$
\begin{aligned}
& u^{(2 k+1)}= \begin{cases}u_{1}^{(2 k+1)}, & \text { in } \Omega_{1}^{\prime} \\
u_{2}^{(2 k)}, & \text { in } \Omega^{\prime} \backslash \Omega_{1}^{\prime},\end{cases} \\
& u^{(2 k+2)}= \begin{cases}u_{1}^{(2 k+1)}, & \text { in } \Omega^{\prime} \backslash \Omega_{2}^{\prime} \\
u_{2}^{(2 k+2)}, & \text { in } \Omega_{2}^{\prime},\end{cases}
\end{aligned}
$$


and $u^{(0)}=\widetilde{u}$, then we have

$$
\begin{array}{ll}
D_{\Omega^{\prime}}\left(u-u^{(2 k+1)}, v_{1}\right)=0, & \forall v_{1} \in V_{1}, \\
D_{\Omega^{\prime}}\left(u-u^{(2 k+2)}, v_{2}\right)=0, & \forall v_{2} \in V_{2} .
\end{array}
$$

Noticing

$$
u^{(2 k+1)}-u^{(2 k)} \in V_{1}, \quad u^{(2 k+2)}-u^{(2 k+1)} \in V_{2}
$$

and

$$
u-u^{(2 k+1)} \in V, \quad u-u^{(2 k+2)} \in V,
$$

Hence,

$$
u^{(2 k+1)}-u^{(2 k)}=P_{V_{1}}\left(u-u^{(2 k)}\right), \quad u^{(2 k+2)}-u^{(2 k+1)}=P_{V_{2}}\left(u-u^{(2 k+1)}\right)
$$

where $P_{V_{i}}: V \rightarrow V_{i}(i=1,2)$ means the projection operator under the inner product $D_{\Omega^{\prime}}(\cdot, \cdot)$ in $V$. Thus (14) is equivalent to

$$
\left\{\begin{array}{l}
u-u^{(2 k+1)}=P_{V_{1}^{\perp}}\left(u-u^{(2 k)}\right), \\
u-u^{(2 k+2)}=P_{V_{2}^{\perp}}\left(u-u^{(2 k+1)}\right) .
\end{array}\right.
$$

Denote the errors as $e_{i}^{(k)}=u-u^{(k)}(i=1,2)$. This leads to

$$
\left\{\begin{array}{l}
e_{1}^{(2 k+1)}=P_{V_{1}^{\perp}} P_{V_{2}^{\perp}} e_{1}^{(2 k-1)}, \\
e_{2}^{(2 k+2)}=P_{V_{2}^{\perp}} P_{V_{1}^{\perp}} e_{2}^{(2 k)},
\end{array}\right.
$$

This implies that, if $\left\{e_{1}^{(2 k+1)}\right\}$ and $\left\{e_{2}^{(2 k)}\right\}$ are convergent, then their limits are in $V_{1}^{\perp} \cap V_{2}^{\perp}$. Similar to the proofs given in $\mathrm{Yu}(1994,2002)$; Luo et al. (2013) we can show the following result.

Theorem 1 There exists a constant $\alpha, 0 \leq \alpha<1$, such that

$$
\left\|e_{1}^{(2 k+1)}\right\|_{1} \leq \alpha^{2 k}\left\|e_{1}^{(1)}\right\|_{1}, \quad\left\|e_{2}^{(2 k+2)}\right\|_{1} \leq \alpha^{2 k+2}\left\|e_{2}^{(0)}\right\|_{1} .
$$

It is obvious to conclude $\alpha$ keeps the convergence of Schwarz alternating method. In the next section, we will prove the contraction factor $\alpha$.

\section{Analysis of the convergence rate}

By Theorem 1, one may find the convergence rate of the above Schwarz alternating algorithm is closely related to the contraction factor $\alpha$, i.e. the overlapping extent of $\Omega_{1}^{\prime}$ and $\Omega_{2}^{\prime}$. Although it can be deduced intuitively that the larger the overlapping part is, the faster convergence rate will be, yet we find it difficult to analyse the convergence rate for general unbounded domain $\Omega^{\prime}$. However, under certain assumptions, we can find out the relationship between contraction factor $\alpha$ and overlapping extent of $\Omega_{1}^{\prime}$ and $\Omega_{2}^{\prime}$. We define three prolate spheroids with the same semi-interfocal distance 


$$
\Gamma_{i}^{\prime}=\left\{(\mu, \theta, \varphi): \mu=\mu_{i}, \theta \in[0, \pi], \varphi \in[0,2 \pi]\right\}, \quad i=0,1,2,
$$

where $\mu_{1}>\mu_{2}>\mu_{0}>0$.

We consider the following boundary value problem over domain $\Omega_{1}^{\prime}$

$$
\begin{cases}-\Delta u=0, & \text { in } \Omega_{1}^{\prime}, \\ u=g_{0}, & \text { on } \Gamma_{0}^{\prime}, \\ u=g_{1}, & \text { on } \Gamma_{1}^{\prime} .\end{cases}
$$

Suppose that

$$
g_{i}(\theta, \varphi)=\sum_{n=0}^{+\infty} \sum_{m=-n}^{n} G_{n m}^{(i)} Y_{n m}(\theta, \varphi), \quad i=0,1
$$

where

$$
G_{n m}^{(i)}=\int_{0}^{\pi} \int_{0}^{2 \pi} g_{i}(\theta, \varphi) Y_{n m}^{*}(\theta, \varphi) \sin (\theta) d \theta d \varphi, \quad i=0,1 .
$$

Then by the separation of variables, we can obtain the solution of (17)

$$
u(\mu, \theta, \varphi)=\sum_{n=0}^{+\infty} \sum_{m=-n}^{n} \frac{\left(S\left(\mu, \mu_{1}\right) G_{n m}^{(0)}+S\left(\mu_{0}, \mu\right) G_{n m}^{(1)}\right)}{S\left(\mu_{0}, \mu_{1}\right)} Y_{n m}(\theta, \varphi)
$$

where $S(x, y)=P_{n}^{m}(\cosh x) Q_{n}^{m}(\cosh y)-P_{n}^{m}(\cosh y) Q_{n}^{m}(\cosh x)$. According to the property of the associated Legendre functions (Gradshteyn and Kyzhik 1980), we have the following lama.

Lemma 1 Let

$$
P_{n}^{m}(x)=\frac{d^{n+m}}{d x^{n+m}}\left(x^{2}-1\right)^{n}
$$

where $n, m$ are both nonnegative integers. If $0 \leq m<n$, then $P_{n m}(x)$ has $n-m$ different zeros $-1=\alpha_{1} \leq \alpha_{2} \leq \cdots \leq \alpha_{n-m}=1$ with $\alpha_{i}=-\alpha_{n-m-(i-1)}, \quad i=1, \ldots, n-m-1$.

Lemma 2 If $\mu>\mu_{0}$, then we conclude

$$
\frac{P_{n}^{m}\left(\cosh \mu_{0}\right)}{P_{n}^{m}(\cosh \mu)}<\left(\frac{\cosh \mu_{0}}{\cosh \mu}\right)^{n},
$$

and

$$
\frac{Q_{n}^{m}(\cosh \mu)}{Q_{n}^{m}\left(\cosh \mu_{0}\right)}<\left(\frac{\cosh \mu_{0}}{\cosh \mu}\right)^{n} .
$$


Proof By the definition of $P_{n}^{m}(x)$ we have

$$
\frac{P_{n}^{m}\left(\cosh \mu_{0}\right)}{P_{n}^{m}(\cosh \mu)}=\left(\frac{\sinh \mu_{0}}{\sinh \mu}\right)^{m-2} \frac{\prod_{i=1}^{n-m}\left(\cosh \mu_{0}-\alpha_{i}\right)}{\prod_{i=1}^{n-m}\left(\cosh \mu-\alpha_{i}\right)} .
$$

For monotonicity, the following holds for $i=1,2, \ldots, n-m$,

$$
\frac{\left(\cosh \mu_{0}-\alpha_{i}\right)\left(\cosh \mu_{0}-\alpha_{n-m-i+1}\right)}{\left(\cosh \mu-\alpha_{i}\right)\left(\cosh \mu-\alpha_{n-m-i+1}\right)}=\frac{\left(\cosh ^{2} \mu_{0}-\alpha_{i}^{2}\right)}{\left(\cosh ^{2} \mu-\alpha_{i}^{2}\right)}<\frac{\cosh ^{2} \mu_{0}}{\cosh ^{2} \mu} .
$$

Hence,

$$
\frac{P_{n}^{m}\left(\cosh \mu_{0}\right)}{P_{n}^{m}(\cosh \mu)}<\left(\frac{\cosh \mu_{0}}{\cosh \mu}\right)^{n} .
$$

On the other hand, (21) can be easily proved by the proposition of Huang and Yu (2006),

Theorem 2 Suppose $g_{0}$ is continuous on $\Gamma_{0}$ and (16) holds. If we apply the Schwarz alternating algorithm given in "Schwarz alternating algorithm based on NBR"section, then

$$
\sup _{\bar{\Omega}_{1}}\left|u-u^{(2 k+1)}\right| \leq C_{1} \alpha^{k}
$$

and

$$
\sup _{\bar{\Omega}_{2}}\left|u-u^{(2 k+2)}\right| \leq C_{2} \alpha^{k+1}
$$

hold true, the constant $C_{i}(i=1,2)$ depend only on $g_{0}$ and $\frac{Q_{n}^{m}\left(\cosh \mu_{i}\right)}{Q_{n}^{m}\left(\cosh \mu_{0}\right)}$ while

$$
0<\alpha=\frac{Q_{n}^{m}\left(\cosh \mu_{1}\right) S\left(\mu_{0}, \mu_{2}\right)}{Q_{n}^{m}\left(\cosh \mu_{2}\right) S\left(\mu_{0}, \mu_{1}\right)}<1 .
$$

Proof Similar to (8), so the solution of the unbounded problem outside of $\Gamma_{0}$ can be expressed as

$$
u(\mu, \theta, \varphi)=\sum_{n=0}^{\infty} \sum_{m=-n}^{n} \frac{Q_{n}^{m}(\cosh \mu)}{Q_{n}^{m}\left(\cosh \mu_{0}\right)} G_{n m}^{(0)} Y_{n m}(\theta, \varphi), \quad \mu \geq \mu_{0} .
$$

Let $\tilde{u}=0$.

By using the algorithm, one has

$$
\begin{aligned}
& u(\mu, \theta, \varphi)-u^{(2 k+1)}(\mu, \theta, \varphi) \\
& =\sum_{n=0}^{+\infty} \sum_{m=-n}^{n} \frac{Q_{n}^{m}\left(\cosh \mu_{1}\right)}{Q_{n}^{m}\left(\cosh \mu_{0}\right)}\left[\frac{Q_{n}^{m}\left(\cosh \mu_{1}\right) S\left(\mu_{0}, \mu_{2}\right)}{Q_{n}^{m}\left(\cosh \mu_{2}\right) S\left(\mu_{0}, \mu_{1}\right)}\right]^{k} \frac{S\left(\mu_{0}, \mu\right)}{S\left(\mu_{0}, \mu_{1}\right)} G_{n m}^{(0)} Y_{n m}(\theta, \varphi),
\end{aligned}
$$

where $\mu_{0} \leq \mu \leq \mu_{1}$. 
By defining

$$
\alpha=\frac{Q_{n}^{m}\left(\cosh \mu_{1}\right) S\left(\mu_{0}, \mu_{2}\right)}{Q_{n}^{m}\left(\cosh \mu_{2}\right) S\left(\mu_{0}, \mu_{1}\right)},
$$

we will show (24).

From Lemma 2, we have

$$
T(\mu)>\left(\frac{\cosh \mu}{\cosh \mu_{0}}\right)^{2 n}>1, \quad \mu>\mu_{0}
$$

and

$$
\frac{T\left(\mu_{1}\right)}{T\left(\mu_{2}\right)}>\left(\frac{\cosh \mu_{1}}{\cosh \mu_{2}}\right)^{2 n}>1,
$$

where $T(\mu)$ is defined as

$$
T(\mu)=\frac{P_{n}^{m}(\cosh \mu) Q_{n}^{m}\left(\cosh \mu_{0}\right)}{P_{n}^{m}\left(\cosh \mu_{0}\right) Q_{n}^{m}(\cosh \mu)} .
$$

Since

$$
\alpha=\frac{T\left(\mu_{2}\right)-1}{T\left(\mu_{1}\right)-1}=1+\frac{T\left(\mu_{2}\right)-T\left(\mu_{1}\right)}{T\left(\mu_{1}\right)-1},
$$

we obtain $0<\alpha<1$. Hence, (22) is accomplished.

Obviously, (23) can be proved with similar process. Finally, the theorem is proved.

Remark The convergence is related on the overlapping part of $\Omega_{1}^{\prime}$ and $\Omega_{2}^{\prime}$. From Theorem 2, we conclude the larger the overlapping part is, the smaller the contraction factor $\alpha$ will be, which identically means the faster the Schwarz alternating algorithm converging.

\section{The error estimates of the algorithm}

Denote $S_{h}\left(\Omega_{1}^{\prime}\right)$ as the linear finite element space over $\Omega_{1}^{\prime}$, where the elements are partitioned as tetrahedrons. Let

$$
\stackrel{\circ}{S}_{h}\left(\Omega_{1}^{\prime}\right)=\left\{v_{h} \in S_{h}\left(\Omega_{1}^{\prime}\right)\left|v_{h}\right|_{\Gamma_{0}^{\prime} \cup \Gamma_{1}^{\prime}}=0\right\} .
$$

$\stackrel{\circ}{h}_{h}\left(\Omega_{1}^{\prime}\right)$ can be regarded as the subspace of $V$ by zero extension. Therefore, we have the discrete Schwarz alternating algorithm as

$$
\begin{cases}\text { Find } w_{1 h}^{(2 k+1)}=u_{1 h}^{(2 k+1)}-\left.u_{h}^{(2 k)}\right|_{\Omega_{1}^{\prime}} \in \stackrel{\circ}{S}_{h}\left(\Omega_{1}^{\prime}\right) & \text { such that } \\ D_{\Omega_{1}^{\prime}}\left(w_{1 h}^{(2 k+1)}, v_{h}\right)=-D_{\Omega_{1}^{\prime}}\left(u_{h}^{(2 k)}, v_{h}\right), & \forall v_{h} \in \stackrel{\circ}{S}_{h}\left(\Omega_{1}^{\prime}\right),\end{cases}
$$

and

$$
\left\{\begin{array}{l}
\text { Find } w_{2 h}^{(2 k+2)}=u_{2 h}^{(2 k+2)}-\left.u_{h}^{(2 k+1)}\right|_{\Omega_{2}^{\prime}} \in V_{2} \quad \text { such that } \\
D_{\Omega_{2}^{\prime}}\left(w_{2 h}^{(2 k+2)}, v\right)=-D_{\Omega_{2}^{\prime}}\left(u_{h}^{(2 k+1)}, v\right), \quad \forall v_{h} \in V_{2}
\end{array}\right.
$$


where

$$
\begin{gathered}
u_{h}^{(2 k+1)}= \begin{cases}u_{1 h}^{(2 k+1)}, & \text { in } \Omega_{1}^{\prime} \\
u_{h}^{(2 k)}, & \text { in } \Omega^{\prime} \backslash \Omega_{1}^{\prime},\end{cases} \\
u_{h}^{(2 k+2)}= \begin{cases}u_{h}^{(2 k+1)}, & \text { in } \Omega^{\prime} \backslash \Omega_{2}^{\prime} \\
u_{2 h}^{(2 k+2)}, & \text { in } \Omega_{2}^{\prime},\end{cases}
\end{gathered}
$$

and $u_{h}^{(0)}=\widetilde{u}$.

By Yu (2002), the solution of (26) can be written as

$$
u_{2 h}^{(2 k+2)}=P \gamma u_{h}^{(2 k+1)},
$$

where $P: H^{\frac{1}{2}}\left(\Gamma_{2}^{\prime}\right) \rightarrow W_{0}^{1}\left(\Omega_{2}^{\prime}\right)$ denotes Poisson integral operator and $\gamma: H^{1}\left(\Omega_{1}^{\prime}\right) \rightarrow H^{\frac{1}{2}}\left(\Gamma_{2}^{\prime}\right)$ denotes trace operator. Combining with (27) and the discrete algorithm, one can easily have the following iteration value:

$$
u_{h}^{(2 k+1)}=\widetilde{u}+ \begin{cases}\sum_{i=0}^{k} w_{1 h}^{(2 i+1)}, & \text { on } \overline{\Omega^{\prime} \backslash \Omega_{2}^{\prime}} \\ \sum_{i=0}^{k} w_{1 h}^{(2 i+1)}+\sum_{j=0}^{k-1}\left[P \gamma w_{1 h}^{(2 j+1)}-w_{1 h}^{(2 j+1)}\right] & \\ \sum_{k}(P \gamma \widetilde{u}-\widetilde{u}), & \text { in } \Omega_{1}^{\prime} \backslash\left(\overline{\Omega^{\prime}} \backslash \Omega_{2}^{\prime}\right), \\ \sum_{j=0}^{k-1} P \gamma w_{1 h}^{(2 j+1)}+\delta_{k}(P \gamma \widetilde{u}-\widetilde{u}), & \text { on } \Omega^{\prime} \backslash \Omega_{1}^{\prime},\end{cases}
$$

and

$$
u_{h}^{(2 k+2)}=\widetilde{u}+ \begin{cases}\sum_{i=0}^{k} w_{1 h}^{(2 i+1)}, & \text { on } \overline{\Omega^{\prime} \backslash \Omega_{2}^{\prime}} \\ \sum_{i=0}^{k} w_{1 h}^{(2 i+1)}+\sum_{j=0}^{k}\left[P \gamma w_{1 h}^{(2 j+1)}-w_{1 h}^{(2 j+1)}\right] & \\ +(P \gamma \widetilde{u}-\widetilde{u}), & \text { in } \Omega_{1}^{\prime} \backslash\left(\overline{\Omega^{\prime}} \backslash \Omega_{2}^{\prime}\right), \\ \sum_{j=0}^{k-1} P \gamma w_{1 h}^{(2 j+1)}+(P \gamma \widetilde{u}-\widetilde{u}), & \text { on } \Omega^{\prime} \backslash \Omega_{1}^{\prime},\end{cases}
$$

where

$$
\delta_{k}= \begin{cases}0, & \text { if } k=0 \\ 1, & \text { if } k>0\end{cases}
$$

The term $\sum_{j=0}^{k-1}$ vanishes at $k=0$. Set

$$
A_{h}\left(\Omega_{2}^{\prime}\right)=\left\{P \gamma\left(v_{h}+\alpha \widetilde{u}+\beta w\right)-\left.\left(v_{h}+\alpha \widetilde{u}+\beta w\right)\right|_{\Omega_{2}^{\prime}} \mid v_{h} \in \stackrel{S}{h}_{h}\left(\Omega_{1}^{\prime}\right), \alpha, \beta \in R, w=u-\widetilde{u}\right\} .
$$

Similarly, we have the $A_{h}\left(\Omega_{2}^{\prime}\right)$ as the subspace of $V$. Hence, $A_{h}\left(\Omega_{2}^{\prime}\right) \subset V_{2} \subset V$. We have the following variational problem on the discrete space 


$$
\begin{cases}\text { Find } v_{h}^{*} \in \stackrel{\circ}{S}_{h}\left(\Omega_{1}^{\prime}\right)+A_{h}\left(\Omega_{2}^{\prime}\right) & \text { such that } \\ D_{\Omega^{\prime}}\left(v_{h}^{*}, v_{h}\right)=-D_{\Omega^{\prime}}\left(\widetilde{u}, v_{h}\right), & \forall v_{h} \in \stackrel{S}{S}_{h}\left(\Omega_{1}^{\prime}\right)+A_{h}\left(\Omega_{2}^{\prime}\right) .\end{cases}
$$

Obviously, the solution of (28) exists uniquely . Set $u_{h}^{*}=v_{h}^{*}+\widetilde{u}$. Similarly in Wu and Yu (2000b), we have the following error estimates.

Theorem 3 For the discrete Schwarz alternating algorithm and the constant $\alpha$ in Theorem 1 , the following error estimates hold

$$
\begin{aligned}
& \left|u-u_{h}^{(2 k+1)}\right|_{1} \leq C h+\alpha^{2 k}\left|u_{h}^{*}-u_{h}^{(1)}\right|_{1}, \\
& \left|u-u_{h}^{(2 k+2)}\right|_{1} \leq C h+\alpha^{2 k+2}\left|u_{h}^{*}-u_{h}^{(0)}\right|_{1} .
\end{aligned}
$$

\section{Numerical results}

Some numerical examples are computed to show the efficiency of our algorithm in this section. Using the method developed in "Schwarz alternating algorithm based on NBR" section. The linear elements is used in the computation of FEM. Computationally, we consider on three meshes: Mesh I, Mesh II and Mesh III. Each mesh is a refinement of its former one, especially as Mesh I is the primary. The refinement is defined as each of elements of the former mesh is divided into eight similar shape equally.

$e$ and $e_{h}$ denote the maximal error of all node functions on $\Gamma_{1 h}$, respectively, i.e.,

$$
\begin{aligned}
& e(k)=\sup _{P_{i} \in \Omega_{1 h}}\left|u\left(P_{i}\right)-u_{1 h}^{(2 k+1)}\left(P_{i}\right)\right|, \\
& e_{h}(k)=\sup _{P_{i} \in \Omega_{1 h}}\left|u_{1 h}^{(2 k-1)}\left(P_{i}\right)-u_{1 h}^{(2 k+1)}\left(P_{i}\right)\right| .
\end{aligned}
$$

$q_{h}(k)$ is the rate of convergence, i.e.

$$
q_{h}(k)=\frac{e_{h}(k-1)}{e_{h}(k)} .
$$

Moreover, we use the relative maximum norm $\left(\left\|E_{u}\right\|_{\infty}\right)$ of the errors between numerical solutions and the exact solutions:

$$
\left\|E_{u}\right\|_{\infty}=\frac{\left|u-u_{h}\right|_{\infty, \Omega_{1}}}{|u|_{\infty, \Omega_{1}}} .
$$

Example 1 Set the cubic $\Omega=\{(x, y, z)|| x|\leq 1| y,|\leq 1| z \mid, \leq 3\}$ and $\Gamma_{0}$ be its surface of $\Omega$. The exact solution of problem (5) be

$$
u=\frac{x / \sqrt{K_{1}}}{\left(\left(x^{2}+y^{2}\right) / K_{1}+z^{2} / K_{2}\right)^{3 / 2}} .
$$

Also $g=\left.u\right|_{\Gamma_{0}}$.

By the theoretical analysis, we take two confocal prolate ellipsoidal surfaces as artificial boundaries, which can be expressed as $\Gamma_{1}=\left\{(\mu, \theta, \varphi) \mid \mu_{1}=1.5, \theta \in[0, \pi], \varphi \in[0,2 \pi]\right\}$ and $\Gamma_{2}=\left\{(\mu, \theta, \varphi) \mid \mu_{2}=1.25, \theta \in[0, \pi], \varphi \in[0,2 \pi]\right\}$. And the semi-interfocal distance 
$f_{1}=f_{2}=6$. Moreover, we have $K_{1}=1$ and $K_{2}=3$. The efficient results are the case in Tables 1, 2 and Fig. 2.

From Table 1, we can see the convergence is really fast. Both $e$ and $e_{h}$ are smaller than them on former mesh. And the Fig. 2 shows us the errors converge rapidly. Both of them reveal that the fine the mesh, the faster the convergence. The numbers of Table 2 testify the remark in "The error estimates of the algorithm" section. By taking different $\mu_{1}$ and $\mu_{2}$, we chose 3 couples of artificial boundaries. Geometrically, the bigger the $\left|\mu_{1}-\mu_{2}\right|$, the bigger the overlapping domain. Within the same triangular partition (Mesh II), we conclude that the bigger the overlapping domain, the faster the convergence.

Example 2 Generally, the $\Omega$ is chosen as a prolate ellipsoidal. Set the semi-interfocal $f_{0}=4$ and $\Gamma_{0}=\left\{(\mu, \theta, \varphi) \mid \mu_{0}=0.5, \theta \in[0, \pi], \varphi \in[0,2 \pi]\right\}$. Set $K_{1}=K_{2}=1$. Thus, the exact solution of problem (5) is

$$
u=\frac{1}{\left(\left(x^{2}+y^{2}\right) / K_{1}+z^{2} / K_{2}\right)^{1 / 2}} .
$$

and $g=\left.u\right|_{\Gamma_{0}}$.

Table 1 The relation between convergence rate and mesh: $\mu_{1}=1.5, \mu_{2}=1.25$

\begin{tabular}{|c|c|c|c|c|c|c|c|}
\hline \multirow[t]{2}{*}{ Mesh } & \multirow[t]{2}{*}{$\mathbf{k}$} & \multicolumn{6}{|c|}{ Number of iteration and corresponding values } \\
\hline & & 0 & 1 & 2 & 3 & 4 & 5 \\
\hline \multirow[t]{3}{*}{ I } & $e$ & $2.4726 \mathrm{E}-1$ & $9.0403 \mathrm{E}-2$ & $5.4826 \mathrm{E}-2$ & $8.0814 \mathrm{E}-3$ & $8.0782 \mathrm{E}-3$ & 8.0774E-3 \\
\hline & $e_{h}$ & - & $2.8013 \mathrm{E}-2$ & $3.6179 E-3$ & $7.2392 \mathrm{E}-4$ & $1.5669 \mathrm{E}-4$ & $3.6362 E-4$ \\
\hline & $q_{h}$ & - & - & 77.4294 & 4.9977 & 4.6200 & 4.3092 \\
\hline \multirow[t]{3}{*}{$\|$} & $e$ & 8.6794E-2 & $4.0215 E-3$ & $3.1259 E-5$ & $2.9243 E-5$ & $2.9104 \mathrm{E}-5$ & $2.9100 E-5$ \\
\hline & $e_{h}$ & - & $1.0366 E-4$ & $3.4624 \mathrm{E}-6$ & $3.1645 E-7$ & 2.8591E-7 & $2.8503 E-7$ \\
\hline & $q_{h}$ & - & - & 29.9437 & 10.9409 & 1.1068 & 1.0031 \\
\hline \multirow[t]{3}{*}{ III } & $e$ & 1.6827E-3 & $9.2546 E-4$ & $7.4972 \mathrm{E}-5$ & $7.4802 \mathrm{E}-5$ & $7.4792 \mathrm{E}-5$ & $7.4753 \mathrm{E}-5$ \\
\hline & $e_{h}$ & - & $9.2858 \mathrm{E}-4$ & $7.6389 E-5$ & $6.6424 \mathrm{E}-6$ & $5.9675 E-6$ & $5.5203 E-6$ \\
\hline & $q_{h}$ & - & - & 12.1564 & 11.5004 & 1.1131 & 1.0817 \\
\hline
\end{tabular}

Table 2 The relation between convergence rate and overlapping degree (Mesh II)

\begin{tabular}{|c|c|c|c|c|c|c|c|c|}
\hline \multirow[t]{2}{*}{$\mu_{1}$} & \multirow[t]{2}{*}{$\mu_{2}$} & \multirow[t]{2}{*}{$k$} & \multicolumn{6}{|c|}{ Number of iteration and corresponding values } \\
\hline & & & 0 & 1 & 2 & 3 & 4 & 5 \\
\hline \multirow[t]{3}{*}{1.5} & 1.2 & $e$ & $6.4728 \mathrm{E}-2$ & $4.6532 \mathrm{E}-3$ & $3.4571 \mathrm{E}-5$ & $2.6119 \mathrm{E}-5$ & $2.6084 \mathrm{E}-5$ & $2.6002 \mathrm{E}-5$ \\
\hline & & $e_{h}$ & - & $2.0222 \mathrm{E}-3$ & $1.2045 E-4$ & $4.5076 \mathrm{E}-5$ & $9.0874 \mathrm{E}-6$ & $9.0244 \mathrm{E}-6$ \\
\hline & & $q_{h}$ & - & - & 16.7890 & 3.8033 & 4.9290 & 1.0660 \\
\hline \multirow[t]{3}{*}{1.5} & 1.0 & $e$ & $4.5186 \mathrm{E}-2$ & $1.0521 \mathrm{E}-3$ & $9.0705 E-5$ & $5.4413 \mathrm{E}-5$ & $1.2218 \mathrm{E}-5$ & $1.2103 E-5$ \\
\hline & & $e_{h}$ & - & $1.3736 \mathrm{E}-3$ & $4.8967 \mathrm{E}-5$ & $2.6640 \mathrm{E}-7$ & $1.4184 \mathrm{E}-7$ & $7.5349 \mathrm{E}-7$ \\
\hline & & $q_{h}$ & - & - & 28.0516 & 18.3810 & 2.7813 & 2.8248 \\
\hline \multirow[t]{3}{*}{1.5} & 0.8 & $e$ & $1.4825 E-3$ & $6.7734 \mathrm{E}-4$ & $9.2125 E-5$ & $1.8249 \mathrm{E}-5$ & $5.6719 \mathrm{E}-6$ & $5.5017 \mathrm{E}-6$ \\
\hline & & $e_{h}$ & - & $6.4936 \mathrm{E}-4$ & $2.1429 E-5$ & $1.2093 \mathrm{E}-6$ & $8.2674 \mathrm{E}-8$ & $1.0827 \mathrm{E}-8$ \\
\hline & & $q_{h}$ & - & - & 30.3022 & 17.7197 & 14.62807 & 7.6359 \\
\hline
\end{tabular}




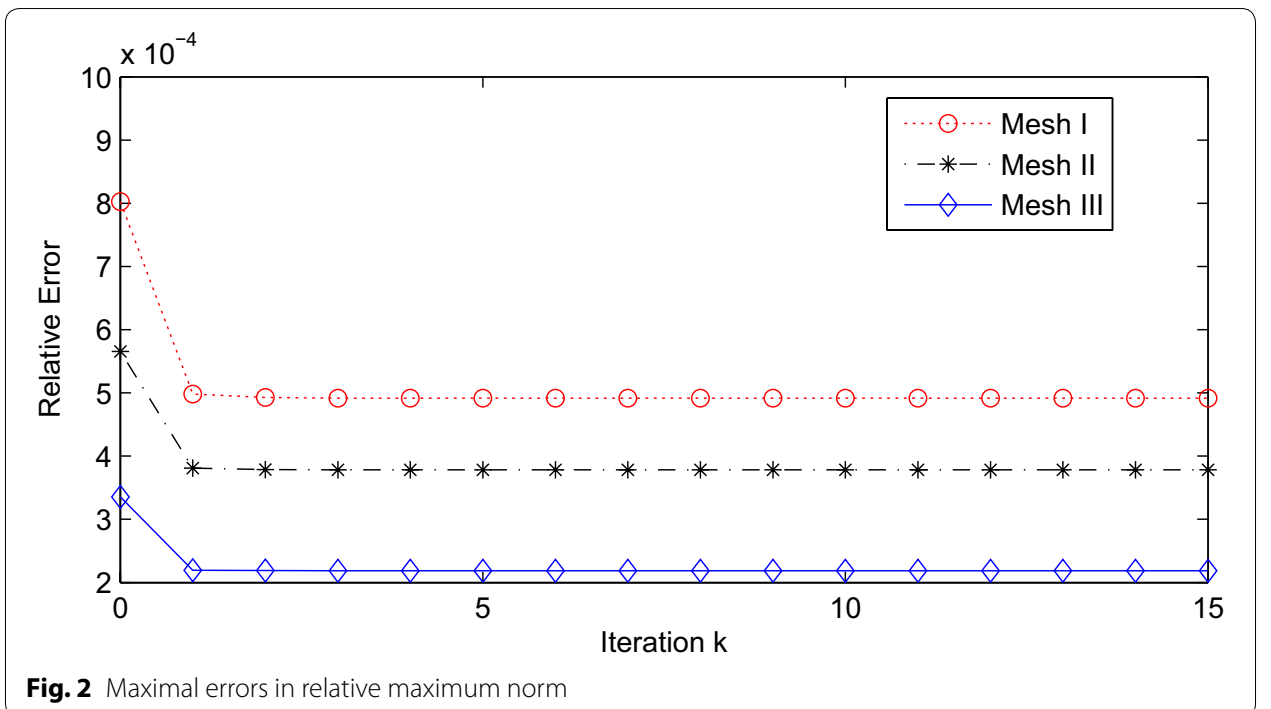

Similarly, we choose two artificial boundaries $\Gamma_{1}$ and $\Gamma_{2}$, which are both confocal with $\Gamma_{0}=\partial \Omega$ as $f_{1}=f_{2}=f_{0}=6$. Let $\Gamma_{1}=\left\{(\mu, \theta, \varphi) \mid \mu_{1}=2.5, \theta \in[0, \pi], \varphi \in[0,2 \pi]\right\}$ and $\Gamma_{2}=\left\{(\mu, \theta, \varphi) \mid \mu_{2}=2.0, \theta \in[0, \pi], \varphi \in[0,2 \pi]\right\}$. The corresponding results are the case in Tables 3, 4 and Fig. 3.

The data of Tables 3 and 4 show us a good convergence. And the analysis of the numbers can be similar to Example 1 .

\section{Conclusions}

In this paper, we construct a Schwarz alternating algorithm for the anisotropic problem on the unbounded domain. The algorithm uses the DDM based on FEM and natural boundary element method. The theoretical analysis shows its convergence is first-order. Further, the rate of convergence is dependent on the overlapping domain. Some numerical examples testify the theoretical conclusions. We can investigate the Schwarz alternating algorithm for anisotropic problem with three different parameters over unbounded domain. Full details and results will be given in a future publication.

Table 3 The relation between convergence rate and mesh: $\mu_{1}=2.5, \mu_{2}=\mathbf{2 . 0}$

\begin{tabular}{|c|c|c|c|c|c|c|c|}
\hline \multirow[t]{2}{*}{ Mesh } & \multirow[t]{2}{*}{$\mathbf{k}$} & \multicolumn{6}{|c|}{ Number of iteration and corresponding values } \\
\hline & & 0 & 1 & 2 & 3 & 4 & 5 \\
\hline \multirow[t]{3}{*}{ 1 } & $e$ & $2.1078 \mathrm{E}-2$ & $8.4562 \mathrm{E}-3$ & $5.9623 \mathrm{E}-3$ & $4.6782 \mathrm{E}-3$ & $4.6511 \mathrm{E}-3$ & $4.6407 \mathrm{E}-3$ \\
\hline & $e_{h}$ & & $9.0022 \mathrm{E}-4$ & $3.0713 \mathrm{E}-5$ & $2.1630 \mathrm{E}-6$ & $1.5593 \mathrm{E}-6$ & $1.1858 \mathrm{E}-6$ \\
\hline & $q_{h}$ & & & 29.3106 & 14.1992 & 1.3871 & 1.3150 \\
\hline \multirow[t]{3}{*}{$\|$} & $e$ & $8.3741 \mathrm{E}-3$ & $7.6501 \mathrm{E}-3$ & $4.6829 \mathrm{E}-3$ & $9.4296 \mathrm{E}-4$ & $8.6241 E-4$ & $8.5788 E-4$ \\
\hline & $e_{h}$ & - & 7.7637E-4 & $1.4383 \mathrm{E}-6$ & $3.7605 E-8$ & $9.6070 E-9$ & $2.4529 E-9$ \\
\hline & $q_{h}$ & - & - & 53.9787 & 38.2471 & 3.9143 & 3.9166 \\
\hline \multirow[t]{3}{*}{ III } & $e$ & $1.8257 \mathrm{E}-3$ & $5.4865 E-4$ & $4.2731 E-5$ & $3.5722 \mathrm{E}-5$ & $3.5605 E-5$ & $3.5592 \mathrm{E}-5$ \\
\hline & $e_{h}$ & - & $1.0350 E-6$ & $5.2502 E-9$ & $1.2387 E-10$ & $3.6938 \mathrm{E}-11$ & $5.0933 \mathrm{E}-11$ \\
\hline & $q_{h}$ & - & - & 197.1280 & 51.8669 & 11.4751 & 6.2403 \\
\hline
\end{tabular}


Table 4 The relation between convergence rate and overlapping degree (Mesh II)

\begin{tabular}{lllllllll}
\hline $\boldsymbol{\mu}_{\mathbf{1}}$ & $\boldsymbol{\mu}_{\mathbf{2}}$ & $\boldsymbol{k}$ & \multicolumn{6}{l}{ Number of iteration and corresponding values } \\
\cline { 4 - 9 } & & & $\mathbf{0}$ & $\mathbf{1}$ & $\mathbf{2}$ & $\mathbf{3}$ & $\mathbf{4}$ & $\mathbf{5}$ \\
\hline 2.5 & 1.8 & $e$ & $7.4537 \mathrm{E}-3$ & $8.6547 \mathrm{E}-4$ & $4.6829 \mathrm{E}-4$ & $9.5781 \mathrm{E}-5$ & $8.7710 \mathrm{E}-5$ & $8.7058 \mathrm{E}-5$ \\
& & $e_{h}$ & - & $6.0775 \mathrm{E}-7$ & $4.7353 \mathrm{E}-8$ & $5.3837 \mathrm{E}-9$ & $6.2859 \mathrm{E}-10$ & $5.6858 \mathrm{E}-10$ \\
& & $q_{h}$ & - & - & 12.8344 & 8.7955 & 8.5647 & 1.1055 \\
\multirow{4}{*}{2.5} & \multirow{2}{*}{1.6} & $e$ & $2.4832 \mathrm{E}-3$ & $7.6489 \mathrm{E}-4$ & $5.4952 \mathrm{E}-5$ & $3.6848 \mathrm{E}-5$ & $2.6981 \mathrm{E}-5$ & $2.6773 \mathrm{E}-5$ \\
& & $e_{h}$ & - & $2.9321 \mathrm{E}-7$ & $1.1713 \mathrm{E}-8$ & $5.8642 \mathrm{E}-10$ & $2.8518 \mathrm{E}-10$ & $2.1763 \mathrm{E}-10$ \\
& & $q_{h}$ & - & - & 25.0324 & 19.9742 & 2.0563 & 1.3104 \\
& \multirow{2}{*}{1.4} & $e$ & $5.4377 \mathrm{E}-4$ & $7.6811 \mathrm{E}-5$ & $6.8129 \mathrm{E}-6$ & $8.1056 \mathrm{E}-7$ & $8.0859 \mathrm{E}-7$ & $8.05378 \mathrm{E}-7$ \\
& & $e_{h}$ & - & $4.2367 \mathrm{E}-7$ & $6.0310 \mathrm{E}-9$ & $1.0814 \mathrm{E}-10$ & $1.9075 \mathrm{E}-11$ & $9.2494 \mathrm{E}-12$ \\
& & $q_{h}$ & - & - & 70.2475 & 55.76912 & 5.6694 & 2.06226 \\
\hline
\end{tabular}

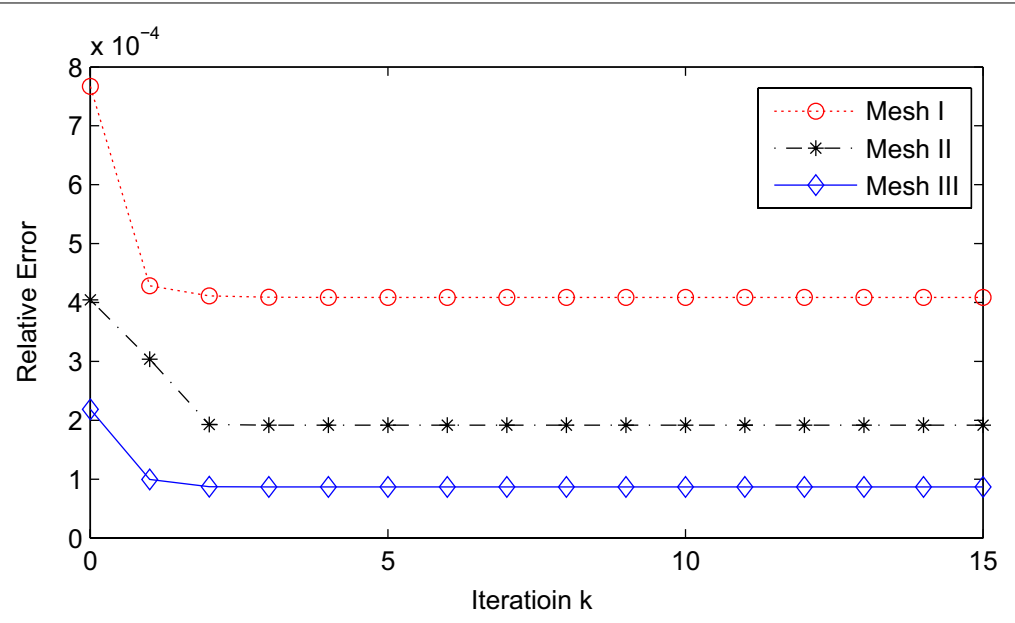

Fig. 3 Maximal errors in relative maximum norm

\section{Authors' contributions}

All authors completed this paper together. All authors read and approved the final manuscript.

\section{Author details}

${ }^{1}$ Jiangsu Key Laboratory for NSLSCS, School of Mathematics Sciences, Nanjing Normal University, No. 1 Wenyuan Road, Nanjing 210023, People's Republic of China. ${ }^{2}$ School of Applied Mathematics, Nanjing University of Finance and Economics, No. 3 Wenyuan Road, Nanjing 210023, People's Republic of China.

\section{Acknowledgements}

All authors are greatly indebted to the referees as the valuable suggestions and comments. This work was subsidized by the National Natural Science Foundation of China (11371198, 11401296), Jiangsu Provincial Natural Science Foundation of China (BK20141008), Natural science fund for colleges and universities in Jiangsu Province (14KJB110007).

\section{Competing interests}

The authors declare that they have no competing interests.

Received: 3 May 2016 Accepted: 12 August 2016

Published online: 26 August 2016

\section{References}

Brenner SC, Scott LR (1996) The mathematical theory of finite element methods. Springer, Berlin Costabel M (1987) Symmetric methods for the coupling of finite elements and boundary elements. In: Brebbia CA (ed) Boundary elements IX, vol 1. Springer, Berlin, pp 411-420 
Das P (2015) Comparison of a priori and a posteriori meshes for singularly perturbed nonlinear parameterized problems. J Comput Appl Math 290:16-25

Das P, Mehrmann V (2016) Numerical solution of singularly perturbed convection-diffusion-reaction problems with two small parameters. BIT Numer Math 56:51-76

Das P, Natesan S (2014) Optimal error estimate using mesh equidistribution technique for singularly perturbed system of reaction-diffusion boundary value problems. Appl Math Comput 249:265-277

Du Q, Yu D (2001) On the natural integral equation for initial boundary value problems of two dimensional hyperbolic equation. Acta Math Appl Sin 24:17-26

Evans D (1977) Numerical solution of exterior problems by the peripheral block over-relaxation method. IMA J Appl Math 19:399-405

Feng K (1980) Differential versus integral equations and finite versus infinite elements. Math Numer Sin 2:100-105

Feng K (1983) Finite element method and natural boundary reduction. In: Proceedings of the international congress of mathematicians, Warszawa, pp 1439-1453

Feng K, Yu DH (1983) Canonical integral equations of elliptic boundary value problems and their numerical solutions. In: Proceedings of China-France symposium on the finite element method. Science Press, Beijing, 1983, pp 211-252

Gatica G, Gatica L, Stephan E (2003) A FEM-DtN formulation for a non-linear exterior problem in incompressible elasticity. Math Methods Appl Sci 26:151-170

Givoli D, Keller J (1989) A finite element method for large domains. Comput Methods Appl Mech Eng 76:41-66

Gradshteyn IS, Kyzhik IM (1980) Table of integrals, series, and products, 8th edn. Academic Press, London

Grote MJ, Keller JB (1995) On non-reflecting boundary conditions. J Comput Phys 122:231-243

Han H (1990) A new class of variational formulations for the coupling of finite and boundary element methods. J Comput Math 8:223-232

Hsiao GC, Porter JF (1986) A hybrid method for an exterior boundary value problem based on asympototic expansion, boundary integral equation and finite element approximations. In: Brebbia CA (ed) Innovative numerical methods in engineering. Springer, Berlin, pp 83-88

Hu Q, Yu D (2001) A solution method for a certain nonlinear interface problem in unbounded domains. Computing 67:119-140 Huang H, Liu D, Yu D (2009) Solution of exterior problem using ellipsoidal artificial boundary. J Comput App Math 231:434-446

Huang H, Yu D (2006) Natural boundary element method for three dimensional exterior harmonic problem with an inner prolate spheroid boundary. J Comput Math 24:193-208

Keller JB, Givoli D (1989) An exact non-reflection boundary conditions. J Comput Phys 82:172-192

Koyama D (2007) Error estimates of the DtN finite element method for the exterior Helmholtz problem. J Comput Appl Math 200:21-31

Koyama D (2009) Error estimates of the finite element method for the exterior Helmholtz problem with a modified DtN boundary condition. J Comput Appl Math 232:109-121

Luo X, Du Q, Huang H, He T (2013) A Schwarz alternating algorithm for a three-dimensional exterior harmonic problem with prolate spheroid boundary. Comput Math Appl 65:1129-1139

MacCamy RC, Marin SP (1980) A finite element method for exterior interface problems. Int J Math Math Sci 3:311-350

Wendland WL (1986) On asymptotic error estimates for combined BEM and FEM. In: Shaw RP (ed) Innovative numerical methods in engineering. Springer, Berlin, pp 55-70

Wu JM, Yu DH (1998) The natural integral equations of 3-D harmonic problems and their numerical solution. Math Numer $\operatorname{Sin} 4: 419-430$

Wu J, Yu D (2000a) The natural boundary element method for exterior elliptic domain. Chin J Num Math Appl 22:91-104

Wu J, Yu D (2000b) The overlapping domain decomposition method for harmonic equation over exterior three-dimensional domain. J Comput Math 18:83-94

Yu D (1994) A domain decomposition method based on the natural boundary reduction over unbounded domain. Math Numer $\operatorname{Sin} 16: 448-459$

Yu D (2002) Natural boundary integral method and its applications, vol 539. Kluwer Academic, Dordrecht

Zhang S, Jin J (1996) Computation of special functions. Wiley, New York

\section{Submit your manuscript to a SpringerOpen ${ }^{\circ}$ journal and benefit from:}

- Convenient online submission

- Rigorous peer review

- Immediate publication on acceptance

- Open access: articles freely available online

- High visibility within the field

- Retaining the copyright to your article

Submit your next manuscript at $\boldsymbol{\nabla}$ springeropen.com 